\title{
Inhibition of prostate cancer RM1 cell growth in vitro by hydroxyapatite nanoparticle-delivered short hairpin RNAs against Stat3
}

\author{
ZUOWEN LIANG ${ }^{1 *}$, HONGLIANG WANG ${ }^{1 *}$, BAOFENG GUO ${ }^{2}$, FUBIAO LI $^{1}$, JINSHA LIU $^{2}$, \\ ZHEWEN LIU ${ }^{3}$, LIBO XU ${ }^{3}$, WENJING YUN ${ }^{3}$, XUEJIAN ZHAO ${ }^{3}$ and LING ZHANG ${ }^{3}$ \\ ${ }^{1}$ Department of Andrology, First Hospital of Jilin University, Changchun, Jilin 130021; \\ ${ }^{2}$ Department of Plastic Surgery, China-Japan Union Hospital of Jilin University, Changchun, Jilin 130031; \\ ${ }^{3}$ Department of Pathophysiology, College of Basic Medicine, Jilin University, Changchun, Jilin 130021, P.R. China
}

Received September 28, 2015; Accepted November 17, 2016

DOI: $10.3892 / \mathrm{mmr} .2017 .6583$

\begin{abstract}
The present study investigated the effect of signal transducer and activator of transcription 3 (Stat3) interference on RM1 prostate cancer cell viability in vitro, using plasmid-based Stat3 specific short hairpin RNA (sh-Stat3) delivered by hydroxyapatite nanoparticles (HAP). HAP carrying sh-Stat 3 plasmids were transfected into tumor cells. MTT assays were used to measure RM1 cell viability 24 and $48 \mathrm{~h}$ following transfection, and the apoptosis rate and cell cycle phase distribution were determined by flow cytometry. Stat 3 mRNA expression levels were measured by reverse transcription-quantitative polymerase chain reaction and Stat3, Cyclin D1, B cell lymphoma 2 apoptosis regulator (Bcl-2), vascular endothelial growth factor (VEGF), Bcl-2 associated $\mathrm{X}$ apoptosis regulator (Bax) and cleaved-caspase- 3 protein expression levels were detected using western blot analysis. The results demonstrated that HAP-delivered sh-Stat 3 significantly decreased RM1 cell viability through the promotion of cell cycle arrest and apoptosis. Stat 3 mRNA and protein expression levels were significantly downregulated in RM1 cells. Bcl-2, VEGF and Cyclin D1 were also significantly downregulated, but cleaved-caspase- 3 and Bax mRNA and protein expression levels were significantly upregulated. HAP-delivered sh-Stat 3 decreased RM1 cell viability in vitro, and HAP assisted plasmid-based delivery of shRNA into tumor cells. The present results suggest that HAP may be a useful method for successful shRNA delivery into tumors.
\end{abstract}

Correspondence to: Dr Ling Zhang, Department of Pathophysiology, College of Basic Medicine, Jilin University, 126 Xinmin Street, Changchun, Jilin 130021, P.R. China

E-mail: zhangling3@jlu.edu.cn

*Contributed equally

Key words: apoptosis, hydroxyapatite nanoparticles, prostate cancer, RNA interference, Stat3

\section{Introduction}

Hydroxyapatite $\left(\mathrm{HA} ; \mathrm{Ca}_{10}\left(\mathrm{PO}_{4}\right)_{6}(\mathrm{OH})_{2}\right)$ is a major component of hard tissues such as tooth and bone (1) and has been used as a biomaterial in multiple applications, including tissue engineering and bone repair (2). HA has been used effectively as a carrier for bioactive molecules, including proteins and DNA, as its biocompatible and porous properties allow it to deliver proteins and genes into cells (3). Previous research has focused on the application of HA as a treatment for bone-associated diseases (4), but research regarding the use of HA as a drug delivery system (DDS) in other tissues remains scarce. One disadvantage associated with the use of HA for DDS is low dispersal in water, making the formulation of a drug-loaded HA treatment of particular importance for development of HA as a DDS. The rise of nanotechnology has provided novel tools and methods for the study of gene carriers. Among these, calcium phosphate nanoparticles have emerged as a vector for nonviral gene delivery $(5,6)$. Hydroxyapatite nanoparticles (HAP) containing pEGFP-N1 plasmids have been reported to transport DNA into gastric cancer cells without any considerable cytotoxicity (7). Tan et al (8) demonstrated that HAP with protamine enhances the efficiency of gene transfection. Sun et al (9) used HAP to deliver the neurotrophin-3 (NT-3) gene into the cochlear neurons of guinea pigs both in vitro and in vivo, and further demonstrated that polyethylenimine-induced surface alteration of HAP permitted specific genetic materials to pass through intact round window membranes in chinchillas with low toxicity and high transfection efficiency (10). Yan-Zhong et al (11) used arginine-modified nanohydroxyapatite to modify the surface charge of HAP and demonstrated an enhanced adsorption capacity in human epithelial cells. $\mathrm{CaCl}_{2}$-modified HAP has also been previously demonstrated to mediate the transfection of small interfering (si-)signal transducer and activator of transcription 3 (Stat3) plasmids into murine prostate cancer cells in vivo, resulting in significant inhibition of cancer growth (12). These previous studies demonstrate that HAP may be a safe and effective gene vector with potential clinical applications. 
RNA interference (RNAi) is a method of post-transcriptional gene silencing. Short hairpin RNA (shRNA) has previously been recognized as a promising novel antitumor strategy (13), but the efficient delivery of shRNA remains a challenge for shRNA-based therapies. Nanomedicine is an emerging field that combines medicine with nanotechnology, and nanoparticles have been used as diagnostic probes in targeted therapies. Hydroxyapatite has been used as a novel biomaterial to treat oral cavities and enhance bone repair, and as a medicine carrier possesses good tissue compatibility both in vivo and in vitro $(14,15)$. Compared with viral vectors, which pose the danger of immunogenicity and pathogenicity, HAP has high osteoconductivity and favorable biocompatibility, and/or osteoinductivity without proinflammatory or immunogenic side effects (16-18). HAP inhibits tumor cell growth directly (19), and is a useful gene delivery system due to its efficiency, safety and economy (12).

DNA vector-based Stat3-specific RNAi (sh-Stat3) silences Stat 3 and inhibits prostate tumor development (13). However, this antitumor activity depends on the efficiency of delivery. The present study examined the effect of hydroxyapatite-transported sh-Stat 3 on the growth of murine prostate cancer cells. A previous study demonstrated that sh-Stat 3 significantly suppresses Stat 3 expression and inhibits prostate cancer cell growth (12). In the present study, HAP was used to deliver DNA-vector-based sh-Stat 3 to treat prostate cancer in vitro, and the in vitro antitumor effects and mechanisms were examined to provide a theoretical basis for future clinical use.

\section{Materials and methods}

Materials. The mouse prostate cancer cell line RM1 was purchased from the Shanghai Institute of Cellular Research (Shanghai, China). The cells were cultured in Iscove's modified Dulbecco's medium (Gibco; Thermo Fisher Scientific, Inc., Waltham, MA, USA) containing 10\% fetal bovine serum (FBS; Gibco; Thermo Fisher Scientific, Inc.), $100 \mathrm{IU} / \mathrm{ml}$ penicillin and $100 \mu \mathrm{g} / \mathrm{ml}$ streptomycin in a humid atmosphere $\left(37^{\circ} \mathrm{C}\right.$, $5 \% \mathrm{CO}_{2}, 95 \%$ air). Plasmids containing sh-Stat3 (sequence GCAGCAGCTGAACAACATG, corresponding to nucleotides 2,144 to 2,162; Genbank accession no. NM_003150) were constructed in situ. According to previous research, the sh-Stat 3 most effective at inhibiting cancer growth is located at the SH2 domain of the mouse and human Stat3 genes (20). A negative control scrambled shRNA sequence (sh-scramble; Ambion; Thermo Fisher Scientific, Inc.) lacking homology to mouse or human gene sequences was used to determine the frequency of nonspecific effects. HAP nanoparticles were purchased from Nanjing Emperor Nano Material Co., Ltd. (Nanjing, China).

Cell culture, transfection and measurement of cell growth. Cell cultures, HAP and transfection plasmids were prepared as previously described (12). For transfection, RM1 cells in the logarithmic growth phase were seeded in 12-well plates ( $1 \times 10^{5}$ cells/well). When the cells reached $50 \%$ confluence, they were transfected with sh-Stat3 (HAP/sh-Stat3 group) or sh-scramble (HAP/sh-scramble group) plasmids, delivered with HAP, for 48-72 $\mathrm{h}$ prior to analysis of mRNA and protein levels, cell apoptosis and viability. As controls, untransfected cells (mock) and cells treated with HAP alone (HAP group) were also used.

Cell viability following plasmid treatment was measured by MTT assay. RM1 cells were seeded in 96-well plates and incubated at $37^{\circ} \mathrm{C}$ for 24 and $48 \mathrm{~h}$ in the presence of sh-Stat 3 or sh-scramble plasmids. Then, $10 \mathrm{ml}$ of MTT (Sigma-Aldrich; Merck Millipore, Darmstadt, Germany) at a concentration of $5 \mathrm{mg} / \mathrm{ml}$ in phosphate buffered saline (PBS) was added to each well, and incubated for $4 \mathrm{~h}$ at $37^{\circ} \mathrm{C}$. Following removal of the culture medium, the formazan crystals were solubilized in $100 \mu 1$ dimethyl sulfoxide (Sigma-Aldrich; Merck Millipore) and shaken for $15 \mathrm{~min}$. Absorption was measured at $570 \mathrm{~nm}$ on a microplate reader (Bio-Rad Laboratories, Inc., Hercules, CA, USA). Each assay was performed nine times.

Apoptosis and cell cycle assessment. RM1 cells $\left(1 \times 10^{6}\right.$ cells per well) were treated with shRNA-Stat 3 and scramble shRNA plasmids for up to $48 \mathrm{~h}$, collected, and resuspended in $100 \mu \mathrm{l}$ PBS, and incubated at room temperature for 5-15 $\mathrm{min}$ in the dark with $5 \mu$ l Annexin V-fluorescein isothiocyanate (eBioscience; Thermo Fisher Scientific, Inc.), then with $5 \mu \mathrm{l}$ propidium iodide (PI; Beckman Coulter, Fullerton, CA, USA) for $30 \mathrm{~min}$ at room temperature in the dark. Flow cytometry was performed to measure apoptosis rates, using an Epics-XL-MCL flow cytometer (Beckman Coulter, Inc.). Data was analyzed using FlowJo v10 software (Tree Star, Inc., Ashland, OR, USA).

Cell cycle distribution was analyzed by measuring the DNA fragments stained with PI. RM1 cells $\left(1 \times 10^{6}\right)$ grown in 6 -well plates were harvested and centrifuged $48 \mathrm{~h}$ following transfection. Cells were counted and washed twice with pre-cooled PBS. Then, cells were fixed and permeabilized overnight by adding $1 \mathrm{ml}$ of $70 \%(\mathrm{v} / \mathrm{v})$ pre-cooled ethanol to each tube at $4^{\circ} \mathrm{C}$. Following centrifugation at $3,000 \mathrm{x} \mathrm{g}$ for $5 \mathrm{~min}$, the fixatives were decanted and $1 \times 10^{6}$ cells were resuspended in $0.5 \mathrm{ml}$ staining solution, containing $50 \mu \mathrm{g} / \mathrm{ml}$ PI and $100 \mu \mathrm{g} / \mathrm{ml}$ DNase-free RNase (Sigma-Aldrich; Merck Millipore) and incubated for $30 \mathrm{~min}$ at room temperature in the dark. Finally, cells were analyzed by flow cytometry using a FACScan $^{\mathrm{TM}}$ system (BD Biosciences, San Jose, CA, USA) and data analysed using CellQuest ${ }^{\mathrm{TM}}$ software (version 3.3; BD Biosciences).

Reverse transcription-quantitative polymerase chain reaction (RT-qPCR) assay. The mRNA expression level of Stat3 and Stat3 downstream genes was determined using semi quantitative RT-PCR. Cells treated with sh-Stat3 or sh-scramble were collected following $48 \mathrm{~h}$ incubation, and total RNA was extracted using TRIzol reagent (Invitrogen; Thermo Fisher Scientific, Inc.). Reverse transcription was performed with $2 \mu \mathrm{g}$ of total RNA using a commercially available RT-PCR kit (cat. no. A4051; Promega Corporation, Madison, WI, USA) according to the manufacturer's instructions, following DNAse I (cat. no. D5025-15KU; Sigma-Aldrich; Merck Millipore) treatment. The primers used for PCR are displayed in Table I. Each reaction ( $20 \mu \mathrm{l}$ samples) was carried out under the following cycling conditions: Initialization for $10 \mathrm{~min}$ at $95^{\circ} \mathrm{C}$ and then 40 cycles of amplification, with $15 \mathrm{sec}$ at $95^{\circ} \mathrm{C}$ for denaturation and $1 \mathrm{~min}$ at $60^{\circ} \mathrm{C}$ for annealing and elongation. A standard curve was plotted for each primer probe, established by using a serial dilution of pooled cDNA from 
Table I. Primer sets used for semi quantitative reverse transcription polymerase chain reaction analysis.

\begin{tabular}{|c|c|c|}
\hline Target gene & Sequence $\left(5^{\prime}-3^{\prime}\right)$ & Accession numbe \\
\hline \multirow[t]{2}{*}{$\beta$-actin } & Forward: CTGAGAGGGAAATCGTGCGT & \\
\hline & Reverse: AACCGCTCGTTGCCAATAGT & NM_007393 \\
\hline \multirow[t]{2}{*}{ Stat3 } & Forward: ACCAGCAATATAGCCGATTCC & \\
\hline & Reverse: CCATTGGCTTCTCAAGATACC & NM_011486 \\
\hline \multirow[t]{2}{*}{$\mathrm{Bcl}-2$} & Forward: TCGCAGAGATGTCCAGTCA & \\
\hline & Reverse: CACCGAACTCAAAGAAGGC & NM_009741 \\
\hline \multirow[t]{2}{*}{ Bax } & Forward: AGGGTTTCATCCAGGATCGAGC & \\
\hline & Reverse: AGGCGGTGAGGACTCCAGCC & NM_007527 \\
\hline \multirow[t]{2}{*}{ Caspase3 } & Forward: TGGACTGTGGCATTGAGAC & \\
\hline & Reverse: AGGAATAGTAACCAGGTGCTG & NM_009810 \\
\hline \multirow[t]{2}{*}{ VEGF } & Forward: ATCTTCAAGCCGTCCTGTG & \\
\hline & Reverse: TGGTGATGTTGCTCTCTGAC & NM_009505 \\
\hline \multirow[t]{2}{*}{ Cyclin D1 } & Forward: TCATTTCCAACCCACCCT & \\
\hline & Reverse: GGCTTCAATCTGTTCCTGG & NM_007631 \\
\hline
\end{tabular}

Stat3, signal transducer and activator of transcription 3; Bcl-2, B cell lymphoma 2 apoptosis regulator; Bax, Bcl-2 associated X apoptosis regulator; VEGF, vascular endothelial growth factor.

cells. All standards and samples were conducted in triplicate, normalised to $\beta$-actin, and relative fold changes in mRNA expression were calculated using the formula $2^{-\Delta \Delta \mathrm{Cq}}(21)$.

Western blot analysis. Cell lysis, protein quantification and western blot assays were performed as previously described (22). Primary antibodies (1:1,000 dilution) targeting Stat3 (cat. no. sc-482), phospho (p-)Tyr705-Stat3 (cat. no. sc-7993), Cyclin D1 (cat. no. sc-450), vascular endothelial growth factor (VEGF; cat. no. sc-152) and $\beta$-actin (cat. no. sc-32251) were obtained from Santa Cruz Biotechnology, Inc. (Dallas, TX, USA). Primary antibodies targeting B cell lymphoma 2 apoptosis regulator (Bcl-2; cat. no. 2876s), Bcl-2 associated $X$ apoptosis regulator (Bax; cat. no. 2772s) and cleaved (c-)caspase3 (cat. no. 9661L) were obtained from Cell Signalling Technology, Inc. (Danvers, MA, USA). Protein bands were visualized using SuperSignal West Pico chemiluminescent substrate (Pierce; Thermo Fisher Scientific, Inc.), and membranes were subjected to X-ray autoradiography. Band intensities were determined with the Quantity One software (v4.2.1; Bio-Rad Laboratories, Inc.). All experiments were performed in triplicate.

Statistical analysis. Quantitative data were expressed as the mean \pm standard error of the mean. Statistical analysis was performed with SPSS version 13.0 (SPSS Inc., Chicago, IL, USA), and one-way analysis of variance followed by Tukey's test was used to compare the differences between groups. $\mathrm{P}<0.05$ was considered to indicate a statistically significant difference.

\section{Results}

Stat3 expression. To calculate the ability of HAP-delivered plasmids to silence Stat 3 expression, RT-qPCR and western blot assays were used to analyze Stat 3 mRNA and protein expression levels, respectively, in RM1 cells. The results demonstrated that Stat3 mRNA expression was significantly decreased in HAP/sh-Stat3-transfected RM1 cells compared with cells transfected with $\mathrm{HAP} / \mathrm{sh}$-scramble $(\mathrm{P}<0.01$; Table II). Stat 3 and $\mathrm{p}$-Stat 3 protein levels were also significantly decreased in HAP/sh-Stat3-transfected RM1 cells compared with RM1 cells transfected with HAP/sh-scramble $(\mathrm{P}<0.01$; Fig. 1).

HAP-delivered sh-Stat3 treatment decreases cell viability in RM1 cells. Cell viability in the HAP/sh-Stat3 group was significantly inhibited compared with the HAP/sh-scramble and Mock groups at $24 \mathrm{~h}(\mathrm{P}<0.01$ and $\mathrm{P}<0.01$, respectively; Fig. 2) and at $48 \mathrm{~h}(\mathrm{P}<0.01$ and $\mathrm{P}<0.01$, respectively; Fig. 2). However, HAP, HAP/sh-scramble and HAP/sh-Stat 3 transfection all reduced cell viability at $48 \mathrm{~h}$ compared with the Mock group; with growth inhibition rates of $12.41 \pm 0.74,13.98 \pm 3.12$ and $44.40 \pm 1.13 \%$, respectively (Fig. 2).

HAP-delivered sh-Stat3 treatment results in G1 arrest and increased apoptosis in RM1 cells. Treatment with HAP-delivered sh-Stat 3 induced significantly increased levels of apoptosis in RM1 cells compared with HAP-sh-scramble cells, HAP-only cells and Mock control cells $(\mathrm{P}<0.01$, $\mathrm{P}<0.01$ and $\mathrm{P}<0.01$, respectively; Fig. 3 and Table III). To determine whether this was associated with specific modifications in the cell cycle, cell cycle analysis was performed in RM1 cells transfected with sh-Stat3 or sh-scramble. $\mathrm{HAP} / \mathrm{sh}$-Stat 3 -treated cells were significantly accumulated in the G1 phase compared with Mock treated cells $(\mathrm{P}<0.01$; Table III), indicating that sh-Stat 3 treatment promotes G1 arrest. Overall, these findings demonstrate that Stat 3 treatment decreases the viability and survival rate of RM1 prostate cancer cells. 
Table II. Reverse transcription-quantitative polymerase chain reaction analysis of Stat3, Bcl-2, Bax, Caspase3, VEGF and Cyclin D1 mRNA expression levels, relative to the Mock group.

\begin{tabular}{lcccccc}
\hline & \multicolumn{7}{c}{ Transcripts } \\
\cline { 2 - 7 } Group (n=3) & Stat3 & Bcl-2 & Bax & Caspase3 & VEGF & Cyclin D1 \\
\hline Mock & 1 & 1 & 1 & 1 & 1 & 1 \\
HAP & $0.84 \pm 0.07$ & $0.77 \pm 0.06$ & $1.03 \pm 0.19$ & $1.49 \pm 0.17$ & $0.96 \pm 0.01$ & $0.41 \pm 0.01^{\mathrm{a}}$ \\
HAP/sh-scramble & $0.58 \pm 0.07$ & $0.14 \pm 0.02^{\mathrm{a}}$ & $1.70 \pm 0.24$ & $3.16 \pm 0.23^{\mathrm{a}}$ & $0.81 \pm 0.02$ & $0.34 \pm 0.01^{\mathrm{a}}$ \\
HAP/sh-Stat3 & $0.25 \pm 0.02^{\mathrm{b}}$ & $0.14 \pm 0.01$ & $4.2 \pm 0.38^{\mathrm{b}}$ & $9.05 \pm 0.74^{\mathrm{b}}$ & $0.11 \pm 0.009^{\mathrm{b}}$ & $0.06 \pm 0.005^{\mathrm{b}}$ \\
\hline
\end{tabular}

${ }^{\text {a }} \mathrm{P}<0.01$ vs. Mock group, ${ }^{\mathrm{b}} \mathrm{P}<0.01$ vs. HAP/sh-scramble. Stat3, signal transducer and activator of transcription 3; Bcl-2, B cell lymphoma 2 apoptosis regulator; Bax, Bcl-2 associated X apoptosis regulator; VEGF, vascular endothelial growth factor; HAP, hydroxyapatite nanoparticles; sh-scramble, scramble short hairpin RNA, sh-Stat3, Stat3 specific short hairpin RNA.
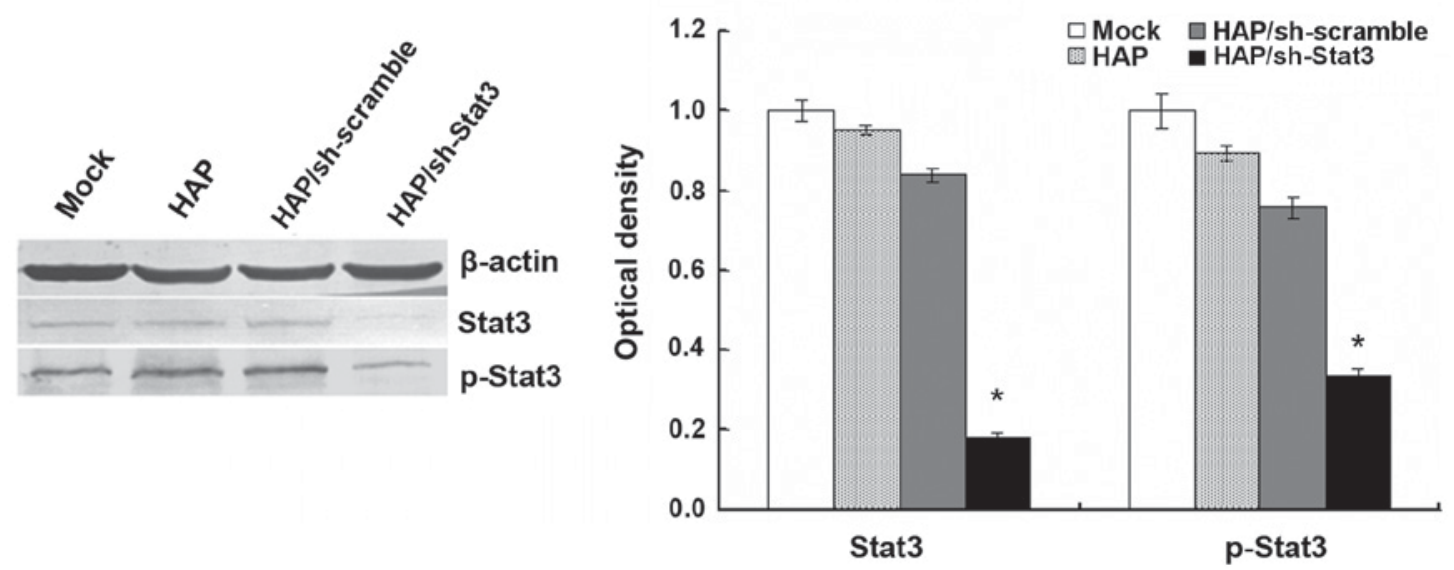

Figure 1. Western blot analysis of Stat3 and p-Stat3 protein in RM1 cells transfected with sh-Stat3, with quantification relative to $\beta$-actin. ${ }^{*} \mathrm{P}<0.01$ vs. scramble control. Stat3, signal transducer and activator of transcription 3; p-, phosphorylated; sh-Stat3, Stat-3 specific short hairpin RNA; HAP, hydroxyapatite nanoparticles; sh-scramble, scramble short hairpin RNA.

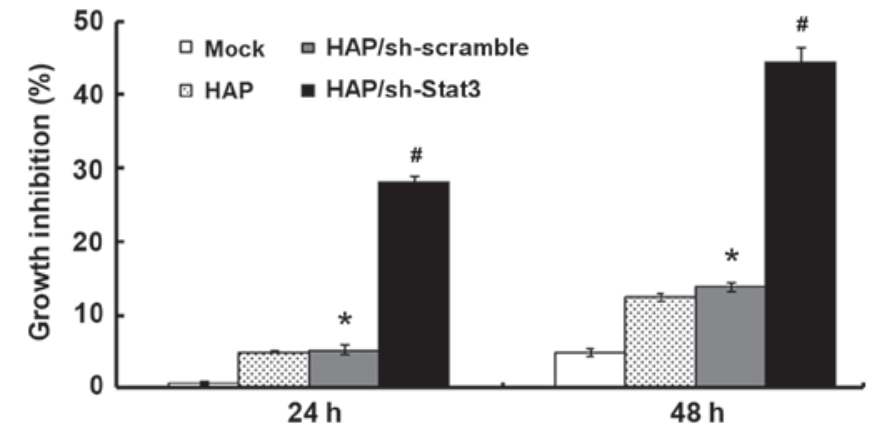

Figure 2. RM1 cell viability in response to transfection with HAP-delivered sh-Stat3, evaluated with MTT assay. Data are presented as the inhibition ratio to $24 \mathrm{~h}$ Mock control. ${ }^{\text {P }}<<0.01$ vs. Mock, ${ }^{\text {"P }}<0.01$ vs. HAP and $\mathrm{HAP} / \mathrm{sh}$-scramble. HAP, hydroxyapatite nanoparticles; sh-Stat3, signal transducer and activator of transcription 3-specific short hairpin RNA; sh-scramble, scramble short hairpin RNA.

Expression levels of Stat3-associated genes. Previous studies have indicated that Stat 3 induces the expression of multiple genes, including the anti-apoptotic protein Bcl-2 and Cyclin D1, which promotes cell division (22). To determine the effect of sh-Stat 3 treatment on the expression of
Stat3-associated genes, RT-qPCR and western blot assays were performed. The results demonstrated that in HAP/sh-Stat3 treated cells compared with Mock treated cells, Bcl-2, VEGF and Cyclin D1 mRNA expression levels were significantly decreased $(\mathrm{P}<0.01, \mathrm{P}<0.01$ and $\mathrm{P}<0.01$, respectively; Table II) and protein expression levels also significantly decreased compared with HAP/sh-scramble treated cells $(\mathrm{P}<0.01, \mathrm{P}<0.01$ and $\mathrm{P}<0.01$, respectively; Fig. 4). However, caspase 3 and Bax levels were significantly increased in HAP/sh-Stat 3 treated cells compared with controls (mRNA expression levels, $\mathrm{P}<0.01$ and $\mathrm{P}<0.01$, respectively; Table II; protein expression levels, $\mathrm{P}<0.01$ and $\mathrm{P}<0.01$, respectively; Fig. 4). These results indicate that treatment with HAP-delivered sh-Stat 3 interferes with the transcriptional activity of Stat 3 and alters the expression of its downstream genes.

\section{Discussion}

Prostate cancer is the second most frequently diagnosed cancer and the sixth leading cause of cancer-associated mortality in men worldwide $(23,24)$. Despite advances in surgical techniques and radiotherapy, prostate cancer continues to pose a medical challenge, with gaining an in-depth understanding of 
Table III. Effect of HAP-delivered sh-Stat3 plasmids on apoptosis and the cell cycle in RM1 cells.

\begin{tabular}{|c|c|c|c|}
\hline Group $(n=3)$ & Apoptotic cells (sum of Q2+Q4 quadrants in Fig. 3) \% & G0-G1, \% & $\mathrm{S}, \%$ \\
\hline Mock & $6.4 \pm 0.98$ & $44.5 \pm 3.14$ & $55.5 \pm 3.14$ \\
\hline HAP & $15.2 \pm 0.74^{\mathrm{a}}$ & $44.4 \pm 2.59$ & $51.6 \pm 3.33$ \\
\hline $\mathrm{HAP} /$ sh-scramble & $17.3 \pm 0.97^{\mathrm{a}}$ & $46.9 \pm 3.95$ & $50.1 \pm 2.58$ \\
\hline $\mathrm{HAP} / \mathrm{sh}-\mathrm{Stat} 3$ & $39.2 \pm 0.74^{\mathrm{b}}$ & $66.7 \pm 3.99^{\mathrm{a}}$ & $24.8 \pm 3.62^{\mathrm{a}}$ \\
\hline
\end{tabular}

${ }^{a} \mathrm{P}<0.01$ vs. mock group, ${ }^{\text {b }}<0.01$ vs. HAP/sh-scramble. Data are presented as the mean \pm standard deviation. HAP, hydroxyapatite nanoparticles; sh-scramble, scramble short hairpin RNA; sh-Stat3, signal transducer and activator of transcription 3 specific short hairpin RNA.
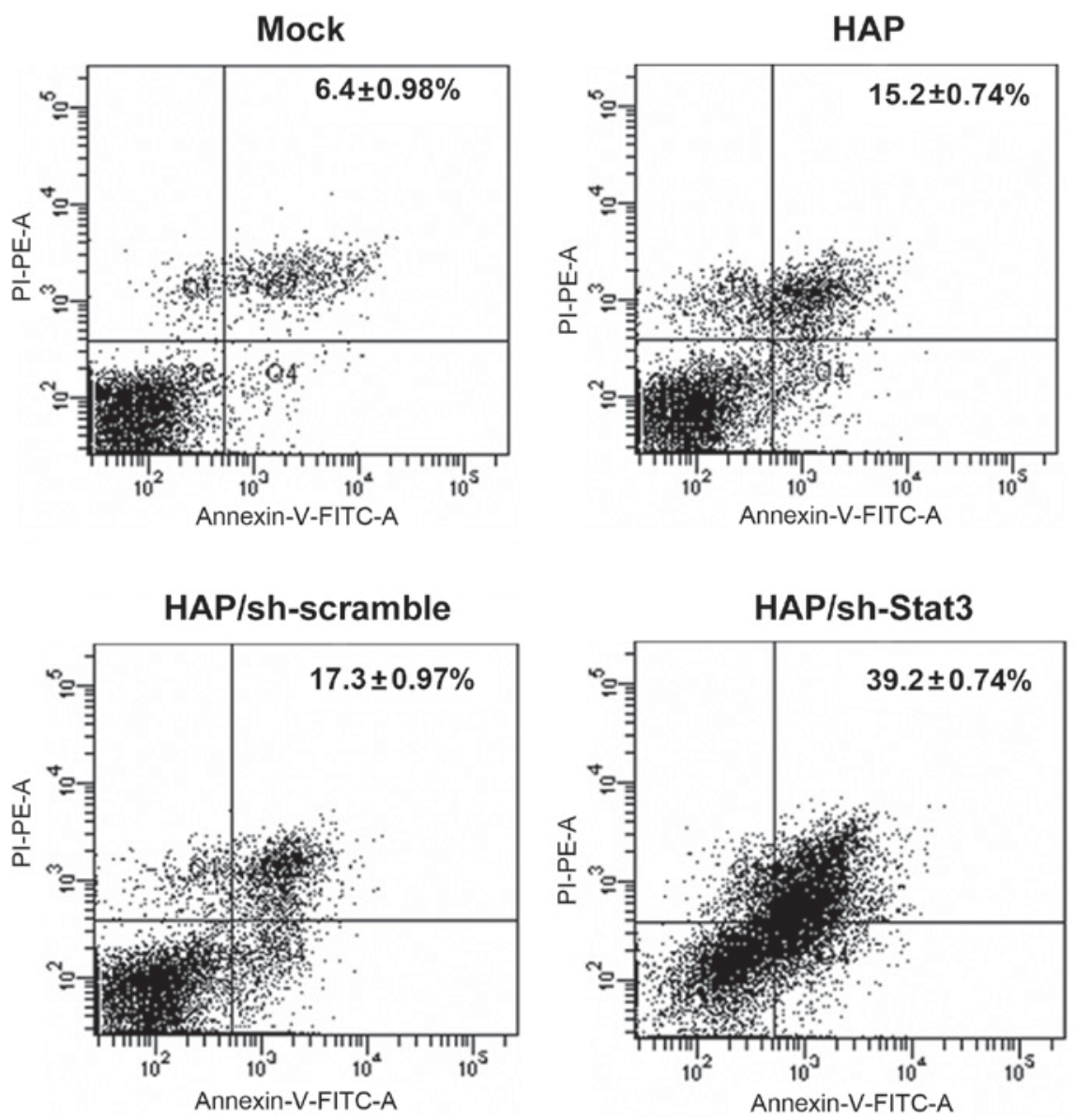

Figure 3. Effect of HAP-delivered sh-Stat 3 treatment on apoptosis in RM1 cells. The bottom right quadrant primarily displays early apoptotic cells $($ Annexin $/ \mathrm{PI})$; the top right quadrant, late apopototic cells $\left(\right.$ Annexin $\left./ \mathrm{PI}^{+}\right)$; the top left quadrant, necrotic cells (Annexin $/ \mathrm{PI}^{+}$); and the bottom left quadrant, live cells (Annexin/PI). HAP, hydroxyapatite nanoparticles; sh-Stat3, signal transducer and activator of transcription 3-specific short hairpin RNA; PI, propidium iodide; FITC, fluorescein isothiocyanate; sh-scramble, scramble short hairpin RNA.

its molecular pathogenesis and the development of novel therapeutic options being of significant importance to global health. As prostate cancer represents an accumulation of genetic mutations in cells and resultant altered function, a potential novel treatment for this cancer is gene therapy (25).

Stat 3 is a transcription factor encoded by the STAT3 gene in humans. It is a member of the STAT protein family, which mediates multiple cellular functions including immunity, proliferation, apoptosis and differentiation. Elevated Stat3 activity has been previously observed in primary tumor tissues and prostate cancer cell lines, and is correlated with tumorigenesis (26). Notably, abnormal Stat3 activation is associated with prostate cancer progression (27). Constitutive activation of Stat3 signalling represents one of the key molecular events in the multistep process of carcinogenesis, and inhibition of Stat3 activation is a potential therapeutic strategy for anti-tumor therapy (28).

In a previous study, DNA vector-based Stat3-specific RNAi was demonstrated to decrease Stat3 signalling (13). However, the efficacy of shRNA-mediated interference relies 

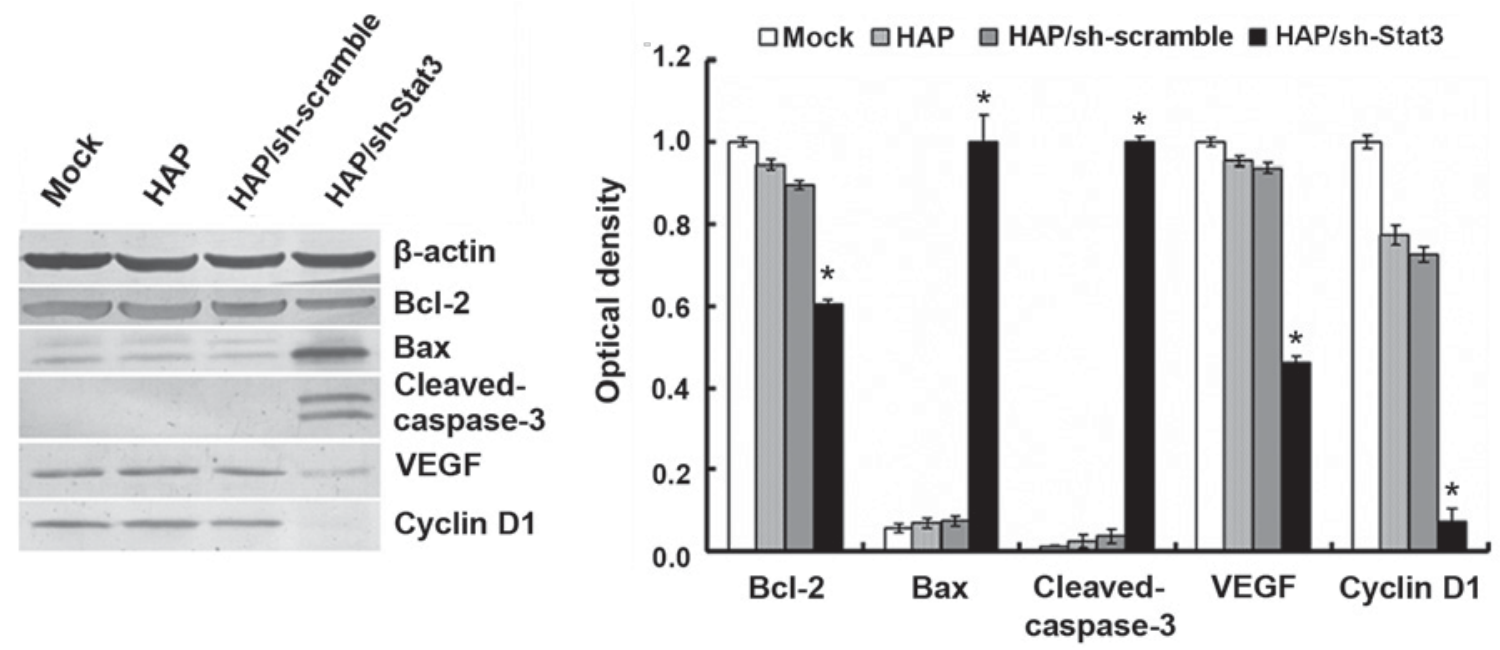

Figure 4. Western blot analysis of Bcl-2, Bax, Caspase3, VEGF and Cyclin D1 protein expression in RM1 cells transfected with HAP-delivered sh-Stat3S "P<0.01 vs. scramble control with quantification relative to $\beta$-actin. Bcl-2, B cell lymphoma 2 apoptosis regulator; Bax, Bcl-2 associated $\mathrm{X}$ apoptosis regulator; VEGF, vascular endothelial growth factor; HAP, hydroxyapatite nanoparticles; sh-Stat3, signal transducer and activator of transcription 3-specific short hairpin RNA; sh-scramble, scramble short hairpin RNA.

on efficient delivery of shRNA oligonucleotides and the major weaknesses that limit most delivery systems' wide application are the potential for oncogenesis or mutagenesis, host immune responses, and high cost (29). Nonviral shRNA delivery does not elicit an immune response, demonstrates improved drug target validation and permits multiple administrations of shRNA, an essential attribute for the therapeutic application of shRNA. Sun et al (9) used HAP as a novel vector for inner ear gene therapy, and previous results have demonstrated that $\mathrm{Ca}^{2+}$ modified HAP mediates sh-Stat 3 plasmid transfection into mouse prostate cancer cells in vivo and in vitro, causing significant inhibition of cancer growth (12). This supported previous reports which demonstrated HAP transfection efficiency reaches $\sim 50-80 \%$ of liposome-mediated transfection (30). The present study revealed that HAP alone exerted antitumor effects: HAP carrying sh-scramble inhibited cancer cell proliferation compared with control, although this inhibition was much stronger when HAP was combined with sh-Stat3. The mechanisms may be related to the intrinsic properties of nanoparticles, and need further research. This result was consistent with the study of Zhu et al (30), in which treatment with HAP alone inhibited hepatocellular carcinoma cell proliferation in vitro. Therefore, the antitumor effect of HAP may be further improved while combined with sh-Stat3.

In the present study, the involvement of Stat 3 in promoting prostate cancer cells proliferation in vitro was confirmed. Stat 3 mRNA and protein expression levels were down-regulated in RM1 cells following sh-Stat 3 treatment, implying that HAP transports sh-Stat 3 into cancer cells, resulting in the inhibition of Stat 3 expression levels. These findings demonstrate that the HAP that carried sh-Stat3 exerts a potent antitumor effect in vitro through decreasing cell viability and the promotion of apoptosis. The data displayed in Table II suggest that inhibition of cancer cell viability was due to a combination of cell cycle arrest and activation of apoptosis. Furthermore, the expression of pro-apoptotic factors including caspase 3 and Bax were significantly upregulated in the sh-Stat 3 group, while anti-apoptotic factors such as Cyclin D1 and Bcl-2 were significantly downregulated. Previous studies have revealed that Stat 3 upregulates several anti-apoptotic proteins' expression, including Bcl-2 and B cell lymphoma-extra large (Bcl-xL) (31), which are key components of mitochondrial apoptotic pathways. Therefore, sh-Stat 3 transfection-induced apoptosis of cancer cells may partially result from the activation of mitochondrial apoptosis pathways (32). Additionally, western blot assays revealed that VEGF expression levels in cancer cells were significantly decreased following sh-Stat3 transfection. Reduced VEGF levels resulting from Stat3 knockdown were expected because VEGF expression has been previously demonstrated to be upregulated by Stat3 (33).

The present study confirmed that HAP is a useful vector for plasmid-based shRNA delivery into cancer cells in vitro, with the proliferation of RM1 cancer cells inhibited by the HAP-delivered sh-Stat3. These findings suggest the potential of HAP as an effective gene delivery vehicle for shRNA-based cancer therapy. However, further modification of HAP is required to enhance transfection efficacy and expedite clinical applications. In conclusion, nanoparticle-mediated sh-Stat3 delivery has potential clinical applications for the treatment of prostate cancers.

\section{Acknowledgements}

The present study was funded by the National Natural Science Foundation of China (grant nos. 81201188 and 81472344).

\section{References}

1. Do TN, Lee WH, Loo CY, Zavgorodniy AV and Rohanizadeh R: Hydroxyapatite nanoparticles as vectors for gene delivery. Ther Deliv 3: 623-632, 2012.

2. Bose $\mathrm{S}$ and Tarafder S: Calcium phosphate ceramic systems in growth factor and drug delivery for bone tissue engineering: A review. Acta Biomater 8: 1401-1421, 2012.

3. Olton D, Li J, Wilson ME, Rogers T, Close J, Huang L, Kumta PN and Sfeir C: Nanostructured calcium phosphates (NanoCaPs) for non-viral gene delivery: Influence of the synthesis parameters on transfection efficiency. Biomaterials 28: 1267-1279, 2007. 
4. Ginebra MP, Canal C, Espanol M, Pastorino D and Montufar EB: Calcium phosphate cements as drug delivery materials. Adv Drug Deliv Rev 64: 1090-1110, 2012.

5. Loo SC, Moore T, Banik B and Alexis F: Biomedical applications of hydroxyapatite nanoparticles. Curr Pharm Biotechnol 11: 333-342, 2010.

6. Chen L, McCrate JM, Lee JC and Li H: The role of surface charge on the uptake and biocompatibility of hydroxyapatite nanoparticles with osteoblast cells. Nanotechnology 22: 105708, 2011.

7. Zakaria SM, Sharif Zein SH, Othman MR, Yang F and Jansen JA Nanophase hydroxyapatite as a biomaterial in advanced hard tissue engineering: A review. Tissue Eng Part B Rev 19: 431-441, 2013.

8. Tan K, Cheang P, Ho IA, Lam PY and Hui KM: Nanosized bioceramic particles could function as efficient gene delivery vehicles with target specificity for the spleen. Gene Ther 14 828-835, 2007.

9. Sun $\mathrm{H}$, Jiang $\mathrm{M}$ and Zhu SH: In vitro and in vivo studies on hydroxyapatite nanoparticles as a novel vector for inner ear gene therapy. Zhonghua Er Bi Yan Hou Tou Jing Wai Ke Za Zhi 43: 51-57, 2008 (In Chinese).

10. Wu X, Ding D, Jiang H, Xing X, Huang S, Liu H, Chen Z and Sun H: Transfection using hydroxyapatite nanoparticles in the inner ear via an intact round window membrane in chinchilla. J Nanopart Res 14: 708, 2012.

11. Yan-Zhong Z, Yan-Yan H, Jun Z, Shai-Hong Z, Zhi-You L and Ke-Chao Z: Characteristics of functionalized nano-hydroxyapatite and internalization by human epithelial cell. Nanoscale Res Lett 6: 600, 2011.

12. Liang ZW, Guo BF, Li Y, Li XJ, Li X, Zhao LJ, Gao LF, Yu H, Zhao XJ, Zhang L and Yang BX: Plasmid-based Stat3 siRNA delivered by hydroxyapatite nanoparticles suppresses mouse prostate tumour growth in vivo. Asian J Androl 13: 481-486, 2011

13. Gao L, Zhang L, Hu J, Li F, Shao Y, Zhao D, Kalvakolanu DV, Kopecko DJ, Zhao X and Xu DQ: Down-regulation of signal transducer and activator of transcription 3 expression using vector-based small interfering RNAs suppresses growth of human prostate tumor in vivo. Clin Cancer Res 11: 6333-6341, 2005.

14. Yamada M, Ueno T, Tsukimura N, Ikeda T, Nakagawa K, Hori N, Suzuki T and Ogawa T: Bone integration capability of nanopolymorphic crystalline hydroxyapatite coated on titanium implants. Int J Nanomedicine 7: 859-873, 2012.

15. Fernandez JM, Molinuevo MS, Cortizo MS and Cortizo AM: Development of an osteoconductive PCL-PDIPF-hydroxyapatite composite scaffold for bone tissue engineering. J Tissue Eng Regen Med 5: e126-e135, 2011.

16. Ye F, Guo H, Zhang H and He X: Polymeric micelle-templated synthesis of hydroxyapatite hollow nanoparticles for a drug delivery system. Acta Biomater 6: 2212-2218, 2010.

17. Liu H, Xi P, Xie G, Chen F, Li Z, Bai D and Zeng Z: Biocompatible hydroxyapatite nanoparticles as a redox luminescence switch. J Biol Inorg Chem 16: 1135-1140, 2011.

18. Barghi L, Asgari D, Barar J, Nakhlband A and Valizadeh $\mathrm{H}$ : Synthesis, characterization and in vitro anti-tumoral evaluation of Erlotinib-PCEC nanoparticles. Asian Pac J Cancer Prev 15: 10281-10287, 2014.
19. Xu J, Xu P, Li Z, Huang J and Yang Z: Oxidative stress and apoptosis induced by hydroxyapatite nanoparticles in $\mathrm{C} 6$ cells. J Biomed Mater Res A 100: 738-745, 2012.

20. Zhang L, Gao L, Li Y, Lin G, Shao Y, Ji K, Yu H, Hu J, Kalvakolanu DV, Kopecko DJ, et al: Effects of plasmid-based Stat3-specific short hairpin RNA and GRIM-19 on PC-3M tumor cell growth. Clin Cancer Res 14: 559-568, 2008.

21. Livak KJ and Schmittgen TD: Analysis of relative gene expression data using real-time quantitative PCR and the 2(-Delta Delta C(T)) Method. Methods 25: 402-408, 2001

22. Barton BE, Karras JG, Murphy TF, Barton A and Huang HF: Signal transducer and activator of transcription 3 (STAT3) activation in prostate cancer: Direct STAT3 inhibition induces apoptosis in prostate cancer lines. Mol Cancer Ther 3: 11-20, 2004.

23. Da Silveira RA, Hermes CL, Almeida TC, Bochi GV, De Bona KS, Moretto MB and Moresco RN: Ischemia-modified albumin and inflammatory biomarkers in patients with prostate cancer. Clin Lab 60: 1703-1708, 2014

24. Dumache R, Puiu M, Motoc M, Vernic C and Dumitrascu V: Prostate cancer molecular detection in plasma samples by glutathione S-transferase P1 (GSTP1) methylation analysis. Clin Lab 60: 847-852, 2014.

25. Torre LA, Bray F, Siegel RL, Ferlay J, Lortet-Tieulent J and Jemal A: Global cancer statistics, 2012. CA Cancer J Clin 65: 87-108, 2015.

26. Aneknan P, Kukongviriyapan V, Prawan A, Kongpetch S, Sripa B and Senggunprai L: Luteolin arrests cell cycling, induces apoptosis and inhibits the JAK/STAT3 pathway in human cholangiocarcinoma cells. Asian Pac J Cancer Prev 15: 5071-5076, 2014.

27. Siveen KS, Sikka S, Surana R, Dai X, Zhang J, Kumar AP, Tan BK, Sethi G and Bishayee A: Targeting the STAT3 signaling pathway in cancer: Role of synthetic and natural inhibitors. Biochim Biophys Acta 1845: 136-154, 2014.

28. Han Z, Wang X, Ma L, Chen L, Xiao M, Huang L, Cao Y, Bai J, Ma D, Zhou J and Hong Z: Inhibition of STAT3 signaling targets both tumor-initiating and differentiated cell populations in prostate cancer. Oncotarget 5: 8416-8428, 2014.

29. Kim WJ and Kim SW. Efficient siRNA delivery with non-viral polymeric vehicles. Pharm Res 26: 657-666, 2009.

30. Zhu SH, Huang BY, Zhou KC, Huang SP, Liu F, Li YM, Xue ZG and Long ZG: Hydroxyapatite nanoparticles as a novel gene carrier. J Nanopart Res 6: 307-311, 2004.

31. Lim SL, Park SY, Kang S, Park D, Kim SH, Um JY, Jang HJ, Lee JH, Jeong CH, Jang JH, et al: Morusin induces cell death through inactivating STAT3 signaling in prostate cancer cells. Am J Cancer Res 5: 289-299, 2014.

32. Zhou Y, Tian L, Zhang YC, Guo BF and Zhou QW: Apoptotic effects of psiRNA-STAT3 on 4T1 breast cancer cells in vitro. Asian Pac J Cancer Prev 15: 6977-6982, 2014.

33. Chen Z and Han ZC: STAT3: A critical transcription activator in angiogenesis. Med Res Rev 28: 185-200, 2008. 